\title{
Chromosome map of the Siamese cobra: did partial synteny of sex chromosomes in the amniote represent "a hypothetical ancestral super-sex chromosome" or random distribution?
}

\author{
Worapong Singchat ${ }^{1,2}$, Rebecca E. O'Connor ${ }^{3}$, Panupong Tawichasri ${ }^{1,2}$, Aorarat Suntronpong ${ }^{1,2}$,
} Siwapech Sillapaprayoon ${ }^{1,2}$, Sunutcha Suntrarachun ${ }^{4}$, Narongrit Muangmai ${ }^{2,5}$, Sudarath Baicharoen ${ }^{6}$, Surin Peyachoknagul ${ }^{7}$, Lawan Chanhome ${ }^{8}$, Darren Griffin ${ }^{3}$ and Kornsorn Srikulnath ${ }^{1,2,9,10^{*}}$ (D)

\begin{abstract}
Background: Unlike the chromosome constitution of most snakes $(2 n=36)$, the cobra karyotype shows a diploid chromosome number of 38 with a highly heterochromatic W chromosome and a large morphologically different chromosome 2. To investigate the process of sex chromosome differentiation and evolution between cobras, most snakes, and other amniotes, we constructed a chromosome map of the Siamese cobra (Naja kaouthia) with 43 bacterial artificial chromosomes (BACs) derived from the chicken and zebra finch libraries using the fluorescence in situ hybridization (FISH) technique, and compared it with those of the chicken, the zebra finch, and other amniotes.

Results: We produced a detailed chromosome map of the Siamese cobra genome, focusing on chromosome 2 and sex chromosomes. Synteny of the Siamese cobra chromosome 2 (NKA2) and NKAZ were highly conserved among snakes and other squamate reptiles, except for intrachromosomal rearrangements occurring in NKA2. Interestingly, twelve BACs that had partial homology with sex chromosomes of several amniotes were mapped on the heterochromatic NKAW as hybridization signals such as repeat sequences. Sequence analysis showed that most of these BACs contained high proportions of transposable elements. In addition, hybridization signals of telomeric repeat $(\text { TTAGGG) })_{n}$ and six microsatellite repeat motifs $\left((A A G G)_{81}(A G A T)_{81}(A A A C)_{81}(A C A G)_{81}(A A T C)_{8,}\right.$ and $(\text { AAAAT) })_{6}$ ) were observed on NKAW, and most of these were also found on other amniote sex chromosomes.

Conclusions: The frequent amplification of repeats might involve heterochromatinization and promote sex chromosome differentiation in the Siamese cobra W sex chromosome. Repeat sequences are also shared among amniote sex chromosomes, which supports the hypothesis of an ancestral super-sex chromosome with overlaps of partial syntenies. Alternatively, amplification of microsatellite repeat motifs could have occurred independently in each lineage, representing convergent sex chromosomal differentiation among amniote sex chromosomes.
\end{abstract}

Keywords: amniote, synteny, repeat sequences, sex chromosome, snake

\footnotetext{
* Correspondence: kornsorn.s@ku.ac.th

Worapong Singchat and Rebecca E. O'Connor are joint first authors.

Darren Griffin and Kornsorn Srikulnath are joint last authors.

'Laboratory of Animal Cytogenetics and Comparative Genomics (ACCG),

Department of Genetics, Faculty of Science, Kasetsart University, Bangkok

10900, Thailand

${ }^{2}$ Animal Breeding and Genetics Consortium of Kasetsart University (ABG-KU),

Bangkok 10900, Thailand

Full list of author information is available at the end of the article
}

(c) The Author(s). 2018 Open Access This article is distributed under the terms of the Creative Commons Attribution 4.0 International License (http://creativecommons.org/licenses/by/4.0/), which permits unrestricted use, distribution, and reproduction in any medium, provided you give appropriate credit to the original author(s) and the source, provide a link to the Creative Commons license, and indicate if changes were made. The Creative Commons Public Domain Dedication waiver (http://creativecommons.org/publicdomain/zero/1.0/) applies to the data made available in this article, unless otherwise stated. 


\section{Background}

Sex chromosomes evolved independently from a homologous autosomal pair in both plants and animals when one chromosomal partner acquired a sex-determining allele $[1,2]$. Differentiated sex chromosomes then developed from the accumulation of sexually antagonistic alleles at loci linked to the sex-specific region. To maintain linkage of these genes, meiotic recombination between proto-sex chromosomes was suppressed around the heterologous region in one sex, which in turn promoted the loss of active genes or segmental insertions and deletions, and caused this region to extend along part or all of the chromosome. This produced chromosomal morphological differences between the $\mathrm{X}$ and $\mathrm{Y}$ (male heterogamety) or $\mathrm{Z}$ and $\mathrm{W}$ (female heterogamety) sex chromosomes. It has also been proposed that structural changes in sex chromosomes might expedite the suppression of recombination, favoring the further amplification of repeat sequences and leading to heterochromatinization [3]. It is thus of great importance to understand the mechanism and role of sex chromosome differentiation with regard to sex determination.

Sex chromosomes of amniotes are highly diverse, ranging from cryptic to highly heteromorphic with $\mathrm{XY}$ or ZW systems. Using comparative gene mapping (chromosome mapping via a cytogenetic technique) and whole -genome sequencing, genomic convergence was discovered, in which unrelated sex chromosomes share syntenies across distantly related taxa [4]. Highly conserved synteny of the chicken $\mathrm{Z}$ chromosome has been found in the $\mathrm{X}$ chromosomes of the Mexican giant musk turtle (Staurotypus triporcatus) and the giant musk turtle (S. salvinii) [5]. However, the X chromosomes of the marsh turtle (Siebenrockiella crassicollis) and wood turtle (Glyptemys insculpta) correspond to chicken chromosome 5, while the $\mathrm{Z}$ chromosome of the Chinese softshell turtle (Pelodiscus sinensis) and the spiny softshell turtle (Apalone spinifera) corresponds to chicken chromosome 15 [6-9]. The Hokou gecko (Gekko hokouenesis, Gekkota) Z chromosome is homologous with the chicken $\mathrm{Z}$ chromosome [10, $11]$, whereas the micro- $X$ chromosome of the Anolis lizard (Anolis carolinensis, Iguania), the micro-Z chromosome of the dragon lizard (Pogona vitticeps, Iguania), and the $\mathrm{Z}$ chromosome of the sand lizard (Lacerta agilis, Lacertoidea) have homology with chicken chromosomes 15, 17 and 23, and 6 and 9, respectively [4, 12-14]. Notably, monotreme sex chromosomes also share partial synteny with the chicken $\mathrm{Z}$ chromosome [15], which corresponds partially to squamate chromosome 2 . Comparative genomics, based on chromosome painting and gene mapping for several squamate reptiles, have revealed that the chicken $\mathrm{Z}$ chromosome is homologous with the short arm of the bi-armed chromosome 2 in most squamate reptiles [11, 13, 14, 16-19]. This suggests the possibility that squamate reptile chromosome 2 is part of a larger ancestral amniote super-sex chromosome with overlaps of partial syntenies among amniotes. However, the hypothesis of an ancestral super-sex chromosome does not just relate to the homology of chicken $\mathrm{Z}$ chromosome, but states that unrelated sex chromosomes share syntenies across distantly related taxa. Ancestral super-sex chromosomes probably exist in amniotes. Multiple translocations have led to the appearance of sauropsid and diapsid sex chromosomes [4].

Snakes (Serpentes), a species-rich lineage of extant reptiles, exhibit conserved ZZ/ZW-type sex chromosomes in most species, except for Boa imperator, Python bivittatus, $P$. regius [20-24]. The $\mathrm{Z}$ chromosome, which is similar in size across species, is the fourth or fifth largest metacentric chromosome, whereas the W chromosome varies from being homomorphic in most henophidians (primitive snakes) to highly differentiated in caenophidians (advanced snakes), as a consequence of different centromere positions and/or amounts of heterochromatin [25-31]. Large amplification of microsatellite repeat motifs or telomeric repeats have been found in the $\mathrm{W}$ chromosome of several colubroid snakes $[25,26]$, and genomic comparisons have revealed that syntenies with the snake sex chromosome are also present in a large variety of chromosomes in squamate reptiles $[4,11-14,16-18,32-34]$. However, the chicken $\mathrm{Z}$ chromosome is homologous with the short arm of snake chromosome 2, as also found in other squamate reptiles, while the snake $\mathrm{Z}$ chromosome is homologous with chicken chromosomes 2 and 27 [21-24, 32-34]. Interestingly, the non-homologous W chromosomes of chickens and the common tiger snake (Notechis scutatus) share common repeat sequences that are not present elsewhere in the genomes analyzed to date [30]. This suggests that repeat sequences are partially shared between the sex chromosomes of the chicken and snake, and supports the hypothesis that squamate chromosome 2 is part of an ancestral super-sex chromosome in amniotes [4].

Unlike most snakes, which have a chromosome number of $2 n=36$, consisting of 16 macrochromosomes and 20 microchromosomes [29, 35], cobra (Naja spp.) karyotypes show $2 \mathrm{n}=38$, comprising five pairs of macrochromosomes and 14 pairs of microchromosomes [35]. In the Siamese cobra (Naja kaouthia), the W chromosome exhibits highly remarkable amplified telomeric repeats, indicating the role of repeat sequences for sex chromosome differentiation. Whereas most snakes have a large submetacentric chromosome 2 , which is also highly conserved in other squamate reptiles, cobras have a large subtelocentric chromosome 2 [35]. Examination of cobra karyotypes points to several fusions and fissions in the Naja lineage, suggesting that the subtelocentric chromosome 2 in the Naja lineage appeared by centric fission of a large submetacentric chromosome 2 in ancestral snake 
karyotypes. By contrast, examples of chromosome 2 resembling those of Naja are not found in the majority of squamate reptiles; however, several examples of lizard subtelocentric/acrocentric chromosome 2 show homology with chromosome 2 of most squamate reptiles [11, 14, 16-19]. An alternative explanation should be considered, whereby the subtelocentric chromosome 2 appeared by intrachromosomal rearrangements in the Naja lineage, although no reports have confirmed this. With these two possible scenarios involving the $\mathrm{W}$ chromosome and structural change in snake chromosome 2 , cobras are very good models with which to study the process of sex chromosome differentiation and evolution, including the hypothesis of an ancestral super-sex chromosome in amniotes. In this study, we constructed a comparative chromosome map with 43 bacterial artificial chromosomes (BACs) derived from the chicken and zebra finch genomes using fluorescence in situ hybridization (FISH) [36-38], and compared the chromosome homology of the Siamese cobra (N. kaouthia) with those of the Japanese striped snake (Elaphe quadrivirgata) and other amniotes. We also screened the Siamese cobra chromosomes using FISH with 19 microsatellite repeat motifs and determined in silico the copy number of microsatellite repeats on the chicken and zebra finch BAC sequences for comparison. This allowed us to delineate the synteny between species, to investigate its significance, and to discuss the hypothesis of an ancestral super-sex chromosome in amniotes.

\section{II ॥ แ}

(4)

a
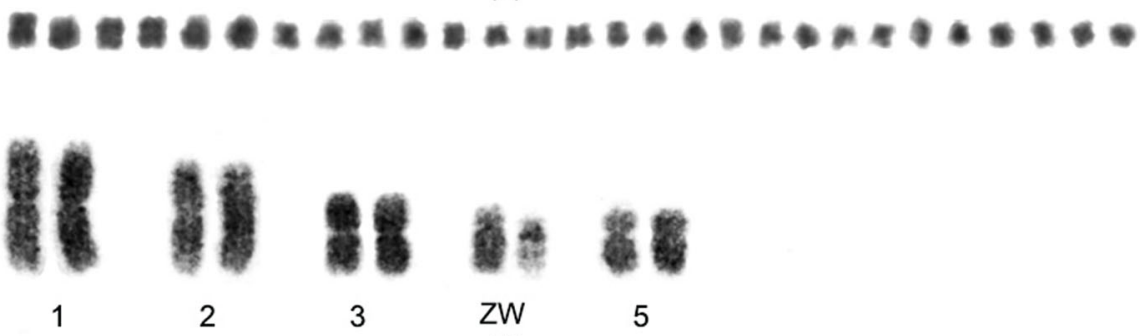

(4)

b

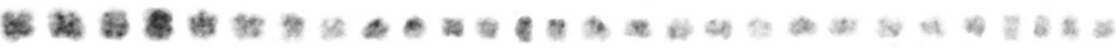
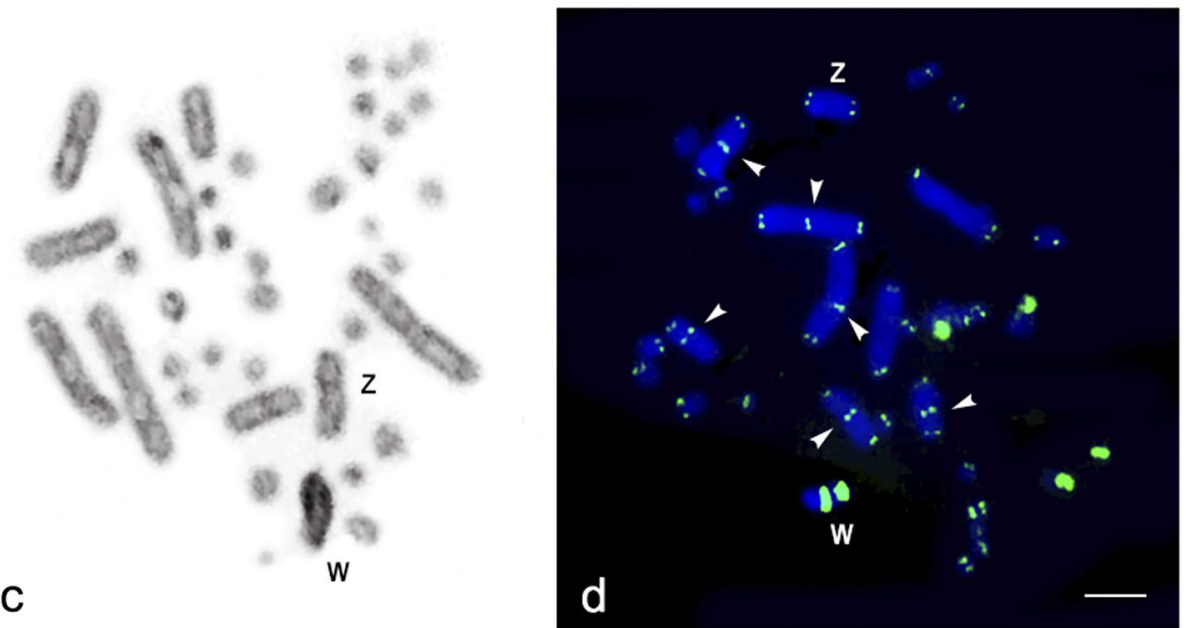

Fig. 1 Karyotype and metaphase spread of the Siamese cobra (Naja kaouthia). Giemsa- (a), grey images of DAPI-stained karyotypes (b), and Cbanded metaphase spread (c) of female Siamese cobra (N. kaouthia). FISH patterns of the telomeric (TTAGGG) nequence on DAPI-stained metaphase chromosome spreads of female Siamese cobra ( $N$. kaouthia) (d). Arrows indicate the centromeric region of chromosome 2 and arrowheads indicate signals of interstitial telomeric sites. "Z" indicates Z sex chromosome, and "W" indicates W sex chromosome. Scale bar represents $10 \mu \mathrm{m}$ 


\section{Results}

Karyotype and Chromosomal Locations of Telomeric Repeat and Microsatellite Repeat Motifs

We examined more than 20 DAPI-stained metaphase spreads from two males and two females. Chromosome numbers were $2 \mathrm{n}=38$, comprising 10 macrochromosomes and 28 microchromosomes in both sexes. The macrochromosomes comprised one pair of large metacentrics (first), one pair of large subtelocentrics (second), two pairs of medium-sized metacentrics (third and fourth), and one pair of submetacentric (fifth). The fourth largest chromosomes were sex chromosomes, in which the female karyotype contained the metacentric $\mathrm{Z}$ chromosome and the submetacentric W chromosome (Figs. 1a and b). The DAPI-stained chromosome banding pattern showed large DAPI-negative bands were on the long arm of the W chromosome, indicating GC richness in this region. $\mathrm{C}$-banding revealed that the $\mathrm{W}$ chromosome exhibited C-positive heterochromatin in the entire region (Fig. 1c). Hybridization signals of hexamer repeat sequences (TTAGGG) $)_{\mathrm{n}}$ were found at the ends of all chromosomes. High intensity signals were observed on two microchromosome pairs. Interstitial telomeric sites were found on three macrochromosome pairs in either sex, while two large-sized (TTAGGG) $)_{n}$ signals on the $q$ arm of the $\mathrm{W}$ chromosome were also observed, but not on the $\mathrm{Z}$ chromosome (Fig. 1d). Eleven of nineteen microsatellite repeat motifs were successfully mapped onto most $N$. kaouthia chromosomes (NKA) (Fig. 2, Additional file 1: Figure S1); however, only six microsatellite repeat motifs showed high amplification on the W chromosome. Strong hybridization signals of tetranucleotide $(\mathrm{AAGG})_{8}$ were localized to NKAWq in addition to the predominant telomeric sites of most chromosomes. By contrast, (AGAT) $(\mathrm{AAAC})_{8},(\mathrm{ACAG})_{8},(\mathrm{AATC})_{8}$, and $(\mathrm{AAAAT})_{6}$ repeat motifs were specifically mapped to NKAWq (Fig. 2).

\section{Chromosome Homology among the Siamese cobra, Chicken, and Zebra finch}

Twenty-six chicken BACs and seventeen zebra finch BACs were mapped to the Siamese cobra chromosomes. We constructed a chromosome map for the Siamese cobra with 43 BACs (markers) (Table 1, Figs. 3 and 4, Additional file 2: Figure S2). More than 20 metaphase spreads were observed for each BAC, with hybridization efficiencies ranging from approximately $70 \%$ to $90 \%$. Chromosome homology between $N$. kaouthia, chicken and zebra finch was analyzed using the chicken genome database (http://www.ncbi.nlm.nih.gov/genome/guide/chicken/) and zebra finch genome database (http://www.ncbi.nlm.nih.gov/genome/367). Seven BACs mapped to NKA2 were localized to GGA1, GGA18 and GGAZ and TGU12 and TGUZ. Three BACs mapped to NKAZ were localized to GGA2p, GGA27, and TGU27. However, twelve BACs on NKAW were located on GGA1, GGA4, GGA9, GGAZ, TGU1B, TGU9, TGU13, TGU17, TGU23, TGU27, and TGUZ. The remaining 22 BACs on NKA1 and microchromosomes were located on GGA1, GGA4, GGA5, GGA9, GGA15, GGA17, GGA23, TGU4A, TGU5, TGU15 and TGU23 (Table 1).
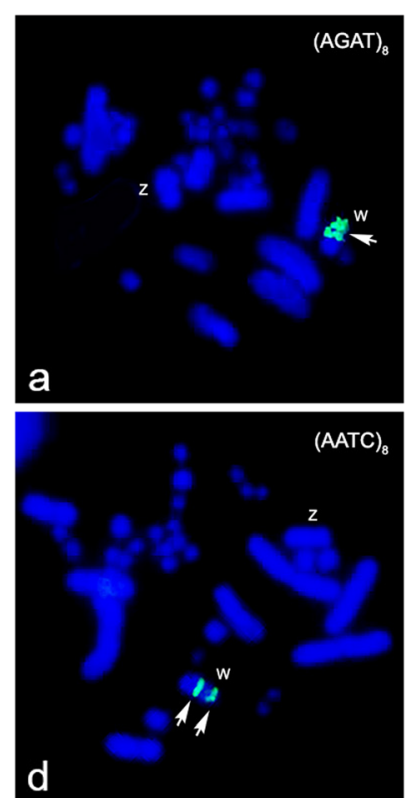

b
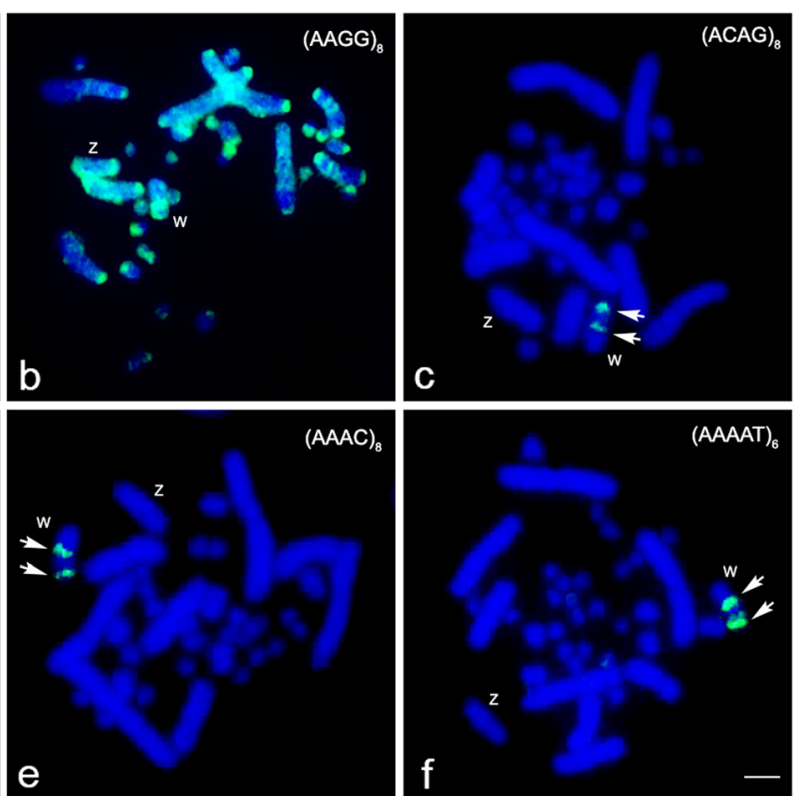

Fig. 2 Chromosomal locations of microsatellite repeat motifs in the Siamese cobra (Naja kaouthia). Hybridization patterns of FITC-labeled (AGAT) 8 $(\mathbf{a})_{,}(\mathrm{AAGG})_{8}(\mathbf{b}),(\mathrm{ACAG})_{8}(\mathbf{c}),(\mathrm{AATC})_{8}(\mathbf{d}),(\mathrm{AAAC})_{8}(\mathbf{e})$, and $(\mathrm{AAAAT})_{6}(\mathbf{f})$ on DAPI-stained chromosomes. Arrows indicate the hybridization signals. " $Z$ " indicates $Z$ sex chromosome, and "W" indicates W sex chromosome. Scale bar represents $10 \mu \mathrm{m}$ 
Table 1 List of chicken and zebra finch BACs mapped to the Siamese cobra chromosomes and their chromosomal location in the chicken (Gallus gallus)

\begin{tabular}{|c|c|c|c|}
\hline Number & $\begin{array}{l}\text { Chicken } \\
\text { chromosome }\end{array}$ & $\mathrm{BAC}$ & $\begin{array}{l}\text { Chromosomal location } \\
\text { in Naja kaouthia }\end{array}$ \\
\hline 1 & 1 & $\mathrm{CH} 261-89 \mathrm{C} 18$ & Wq \\
\hline 2 & 1 & $\mathrm{CH} 261-184 \mathrm{E} 5$ & micro \\
\hline 3 & 1 & $\mathrm{CH} 261-125 \mathrm{~F} 1$ & $2 q$ \\
\hline 4 & 1 & TGMCBA-167P13 & Wq \\
\hline 5 & 1 & CH261-36B5 & micro \\
\hline 6 & 1 & CH261-18J16 & micro \\
\hline 7 & 1 & $\mathrm{CH} 261-83 \mathrm{O} 13$ & micro \\
\hline 8 & $2 p$ & $\mathrm{CH} 261-177 \mathrm{~K} 1$ & Z \\
\hline 9 & 4 & $\mathrm{CH} 261-71 \mathrm{~L} 6$ & Wq \\
\hline 10 & 4 & CH261-18C6 & $1 \mathrm{q}$ and micro \\
\hline 11 & 4 & $\mathrm{CH} 261-85 \mathrm{H} 10$ & micro \\
\hline 12 & 4 & TGMCBA-200G5 & micro \\
\hline 13 & 4 & TGMCBA-280M7 & micro \\
\hline 14 & 4 & TGMCBA-330J11 & micro \\
\hline 15 & 5 & $\mathrm{CH} 261-2 \mathrm{I} 23$ & $1 q$ \\
\hline 16 & 5 & TGMCBA-145C6 & $1 q$ \\
\hline 17 & 5 & $\mathrm{CH} 261-49 \mathrm{~B} 22$ & 1q and micro \\
\hline 18 & 9 & $\mathrm{CH} 261-95 \mathrm{~N} 3$ & Wq \\
\hline 19 & 9 & TGMCBA-217A3 & Wq \\
\hline 20 & 9 & $\mathrm{CH} 261-183 \mathrm{~N} 19$ & micro \\
\hline 21 & 9 & $\mathrm{CH} 261-187 \mathrm{M} 16$ & micro \\
\hline 22 & 12 & TGMCBA-305E19 & $2 q$ \\
\hline 23 & 13 & TGMCBA-136|12 & Wq \\
\hline 24 & 15 & $\mathrm{CH} 261-90 \mathrm{P} 23$ & micro \\
\hline 25 & 15 & TGMCBA-266G23 & micro \\
\hline 26 & 17 & TGMCBA-37515 & Wq \\
\hline 27 & 17 & TGMCBA-67H23 & Wq \\
\hline 28 & 17 & $\mathrm{CH} 261-69 \mathrm{M} 11$ & micro \\
\hline 29 & 18 & $\mathrm{CH} 261-60 \mathrm{~N} 6$ & $2 q$ \\
\hline 30 & 18 & CH261-67N15 & $2 q$ \\
\hline 31 & 18 & $\mathrm{CH} 261-72 \mathrm{~B} 18$ & $2 q$ \\
\hline 32 & 23 & TGMCBA-227A15 & Wq \\
\hline 33 & 23 & $\mathrm{CH} 261-191 \mathrm{G} 17$ & micro \\
\hline 34 & 23 & $\mathrm{CH} 261-105 \mathrm{P} 1$ & micro \\
\hline 35 & 23 & CH261-49G9 & micro \\
\hline 36 & 23 & TGMCBA-173N15 & micro \\
\hline 37 & 23 & TGMCBA-48O8 & micro \\
\hline 38 & 27 & TGMCBA-23C5 & $\mathrm{Zq}$ and $\mathrm{Wq}$ \\
\hline 39 & 27 & $\mathrm{CH} 261-66 \mathrm{M} 16$ & Z \\
\hline
\end{tabular}

Table 1 List of chicken and zebra finch BACs mapped to the Siamese cobra chromosomes and their chromosomal location in the chicken (Gallus gallus) (Continued)

\begin{tabular}{llll}
\hline Number & $\begin{array}{l}\text { Chicken } \\
\text { chromosome }\end{array}$ & BAC & $\begin{array}{l}\text { Chromosomal location } \\
\text { in Naja kaouthia }\end{array}$ \\
\hline 40 & $Z$ & CH261-129A16 & Wq \\
41 & $Z$ & TGMCBA-200J22 & Wq \\
42 & $Z$ & TGMCBA-27019 & $2 q$ \\
43 & $Z$ & CH261-133M4 & $2 q$ \\
\hline
\end{tabular}

\section{Sequence Analysis of Chicken and Zebra finch BACs Located on the Siamese cobra W sex chromosomes}

The content of repeat sequences in chicken and zebra finch BACs mapped to NKAW was examined using Repbase-GIRI and MSATCOMMANDER (Tables 2 and 3). All BACs contained 3.04-24.85\% repeat sequences, of which $0.07-1.26 \%$ were transposable elements. The BACs mapped to NKAW tended to have twice as many repeat sequences as BACs mapped to NKAZ, NKA2, or other chromosomes (Additional files 3, 4, 5, 6, 7 and 8: Tables S1, S2, S3, S4, S5, and S6), showing no preferential accumulation of any specific repeat sequences. Our analysis identified several microsatellite repeat motifs within BACs mapped to NKAW, most of which were not the same as those found using microsatellite repeat motif mapping (Table 3, Fig. 2). Additionally, BLAST analysis of BAC sequences mapped to NKAW revealed the predictions of five functional genes, including $D D X 26 B, D H X 36, F H L 1$, GPR149, and MAP7D3, which were related to sex determination and sex development in vertebrates (Table 4).

\section{Discussion}

Comparison of the draft genome assembly and chromosome maps among amniotes revealed a high level of conservation for synteny and allows us to deduce the process of chromosomal rearrangement over millions of years [11-14, 16-18, 21, 33, 34]. However, sex chromosomes are a more dynamic entity in the genome than autosomes [3, 39]. The $\mathrm{W}$ chromosome of snakes is the most variable element in the snake genome, with differences in the number of genes and amplification of several repeat motifs, even between closely related species $[21,22,25,26]$. This suggests that molecular differentiation has often occurred on the W chromosome in snakes, after divergence from a common ancestor. Amplification of repeat sequences might also have resulted in the large size of the $\mathrm{W}$ chromosome found in the tiger snake (N. scutatus) and the tiger keelback snake (Rhabdophis tigrinus), arising from a recent addition of repeats $[26,30,40]$. Conversely, the small size of the W chromosome in the Japanese striped snake (E. quadrivirgata) may be a consequence of degradation [21]. 

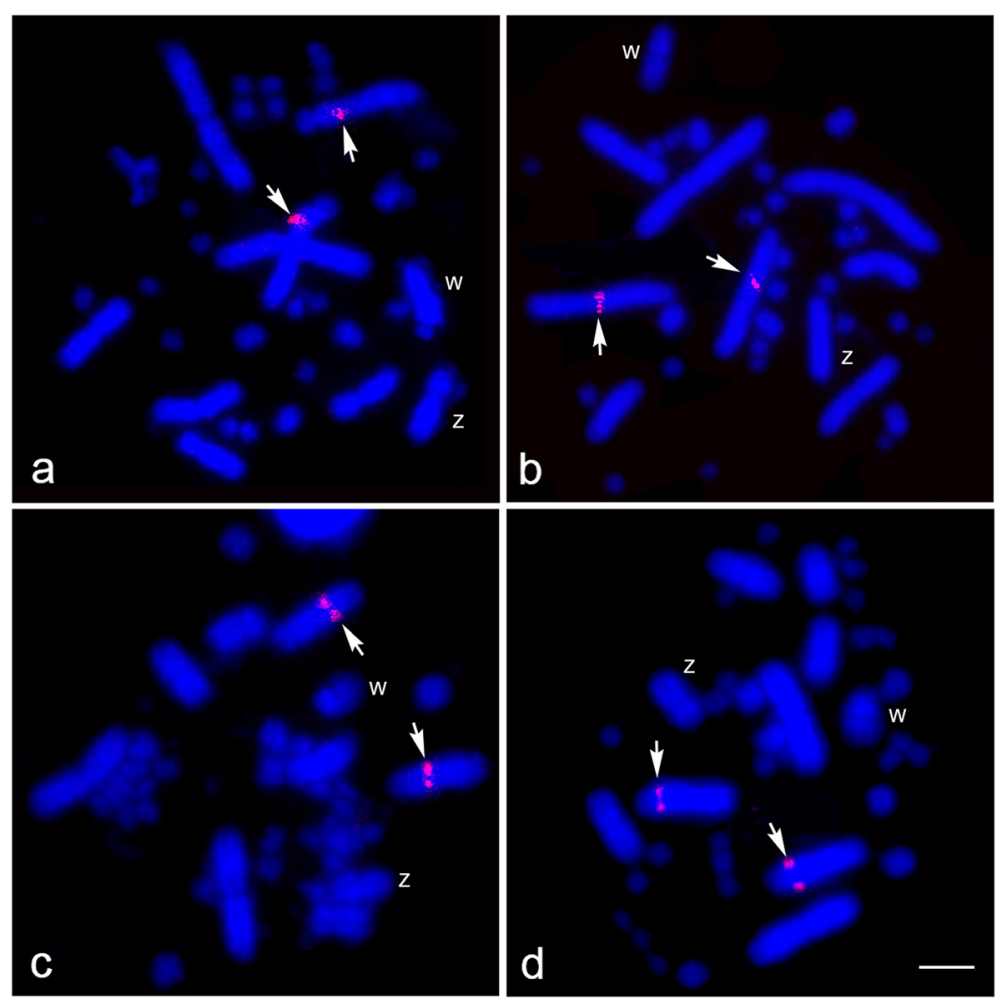

Fig. 3 Chromosomal locations of chicken and zebra finch BACs in female Siamese cobra (Naja kaouthia). GGAZ BAC (CH261-133M4) (a), GGA18 BAC (CH261-67N15) (b), GGA18 BAC (CH261-72B18) (c), and GGA12 BAC (TGMCBA-305E19) (d) were localized to chromosome 2 (NKA2). Arrows indicate the hybridization signals. "Z" indicates Z sex chromosome, and "W" indicates W sex chromosome. Scale bar represents $10 \mu \mathrm{m}$

\section{Amplification of Microsatellite Repeat Motifs on the Siamese cobra W Chromosome}

The $\mathrm{W}$ chromosome was smaller than the Z chromosome in the Siamese cobra, while C-banding revealed almost the entire heterochromatic nature of the W chromosome. This suggests that size reduction and heterochromatinization are correlated with W chromosome differentiation in the Siamese cobra. Our FISH analyses revealed that six microsatellite repeat motifs $\left((\mathrm{AAGG})_{8}\right.$, $(\mathrm{AGAT})_{8},(\mathrm{AAAC})_{8},(\mathrm{ACAG})_{8},(\mathrm{AATC})_{8}$, and (AAAA $\mathrm{T})_{6}$ ) were amplified on NKAWq. Hybridization signals of six microsatellite repeat motifs also overlapped with telomeric (TTAGGG) $)_{\mathrm{n}}$ repeats identified on the NKAWq. These results collectively suggest that the NKAW are structural complexes containing various repeat sequences on the female-specific region. A different hybridization pattern among repeat motifs indicates that each repeat type is distinct. Such amplification of microsatellite repeats and telomeric repeats in the heterochromatic region on $\mathrm{W}$ chromosomes has been reported in several caenophidian snakes and other squamate reptiles [25-27, 41] (Fig. 5). One microsatellite repeat motif amplified on the $\mathrm{W}$ chromosome in several caenophidian snakes though not for basal lineage is the banded krait minor satellite $(\mathrm{Bkm})$, consisting of microsatellite repeat motif $(A G A T)_{n}$ or $(G A C A)_{n}$ sequences, and related to the degree of ZW differentiation [25, 26, 42, 43]. This also suggests that frequent amplification of the repeats has a structural role in heterochromatinization and promotes further sex chromosome differentiation.

Amplification of (ACAG) $)_{n}$ and (AATC) $)_{n}$ was found on the W chromosome of tiger snakes ( $N$. scutatus), while $(\mathrm{AGAT})_{\mathrm{n}}$ was also observed in the Japanese striped snake (E. quadrivirgata), and the tiger keelback snake ( $R$. tigrinus) [26] (Fig. 5). However, the sets of microsatellite repeat motifs used in this study generally differed from those in previous reports. Therefore, it cannot be ruled out that other microsatellite repeat motifs might be amplified in the Siamese cobra. Interestingly, both (AAGG) and telomeric repeats identified on the central bearded dragon (P. vitticeps) W chromosome were also identified on NKAW, even though the species are only distantly related ([26, 41, 44]; Srikulnath et al. unpublished data). Similar results were also found in other amniotes (Fig. 5). This suggests that amplification of microsatellite repeat motifs occurred independently in each lineage and might represent convergent sex chromosomal differentiation among amniote sex chromosomes. It should be 

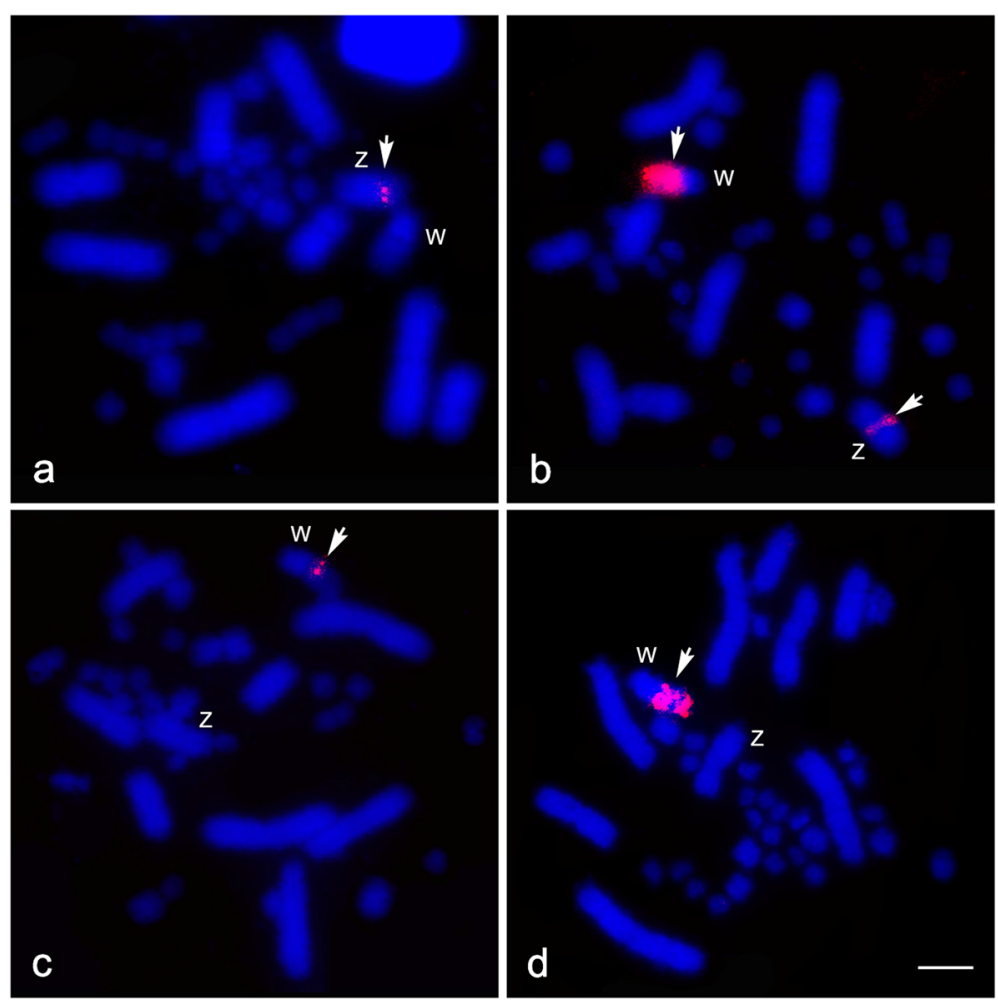

Fig. 4 Chromosomal locations of chicken and zebra finch BAC clones in female Siamese cobra (Naja kaouthia). GGA2p BAC (CH261-177K1) (a), TGU27 BAC (TGMCBA-23C5) (b), GGA9 BAC (CH261-95N3) (c), and GGAZ BAC (CH261-129A16) (d) were localized to W chromosome (NKAW). Arrows indicate the hybridization signals. "Z" indicates Z sex chromosome, and "W" indicates W sex chromosome. Scale bar represents $10 \mu m$

considered that although it is known that sex chromosomes share no homology among amniotes, evidence of synteny from several amniotes showed some overlap of partial synteny likely to have been part of an ancestral super-sex chromosome [4]. An example of such non-homologous W chromosomes of the chicken and snake sharing several repeat groups has been found in the common tiger snake (N. scutatus) [30]. Is it possible that amplified microsatellite repeat motifs were retained in the sex chromosomes of a common ancestor and subsequent reshuffling provided the appearance of sex chromosomes in each lineage?

\section{Is squamate reptile chromosome 2 part of an ancestral} super-sex chromosome in amniotes?

Except for gecko lizards and lacertid lizards, synteny on the snake $\mathrm{Z}$ chromosome and chromosome 2 are remarkably well conserved in most squamate reptiles [11-14, 16-18, 30]. Comparison of the chromosome maps of the Siamese cobra and other squamate reptiles revealed that subtelocentric NKA2 was homologous with submetacentric chromosome 2 of most squamate reptiles [11, 14, 16-19, 21]. This suggests that NKA2 resulted from intrachromosomal rearrangements (such as pericentric inversion or centromere repositioning) in an ancestral bi-armed macro chromosome. However, large hybridization signals like repeat sequences of one GGAZ BAC (CH261-129A16) and one TGUZ BAC (TGMCBA-200J22) observed on the Siamese cobra W chromosome were not present in other Siamese cobra chromosomes, even though these two BACs were expected to be located on NKA2. Similar results were also found in an additional eight BACs derived from GGA1, GGA4, TGU1B, TGU13, TGU17, TGU23, TGU27, which showed partial homology with various amniote sex chromosomes (Fig. 6) [4]. This suggests that these BACs contained repeat sequences shared with NKAW. Sequence analysis using RepeatMasker and MSATCOMMANDER showed $1.95-23.26 \%$ of repeat sequences for retrotransposon and $0.58-1.66 \%$ for microsatellites repeats, also found in BACs mapped on NKAW. Transposable elements form the majority of shared elements in NKAW, supporting the co-location of heterochromatin and putative transposable elements as a result of the unchecked activity of retrotransposons in cells [45]. These repeat sequences probably drive these regions as derived sex chromosomes [3]. In agreement with the hypothesis of an ancestral amniote super-sex chromosome, observations of hybridization patterns from chicken and zebra finch BACs mapped to 
Table 2 Comparison of major classes of repeat sequences in chicken and zebra finch BACs mapped on the Siamese cobra W chromosome

\begin{tabular}{|c|c|c|c|c|c|c|c|c|c|c|c|c|}
\hline & $\mathrm{NE}$ & LO & $\%$ & $\mathrm{NE}$ & LO & $\%$ & $\mathrm{NE}$ & LO & $\%$ & $\mathrm{NE}$ & LO & $\%$ \\
\hline & \multicolumn{3}{|c|}{$1 \mathrm{CH} 261-89 \mathrm{C} 18$} & \multicolumn{3}{|c|}{1 TGMCBA-167P13 } & \multicolumn{3}{|c|}{4 CH261-71L6 } & \multicolumn{3}{|c|}{$9 \mathrm{CH} 261-95 \mathrm{~N} 3$} \\
\hline$\% G C$ & & & 47.61 & & & 59.52 & & & 44.85 & & & 42.81 \\
\hline Satellites & 2 & 580 & 0.34 & 1 & 19 & 0.02 & 0 & 0 & 0 & 0 & 0 & 0 \\
\hline Simple repeats & 30 & 988 & 0.58 & 23 & 2011 & 1.91 & 36 & 1910 & 1.02 & 70 & 2777 & 1.43 \\
\hline Retroelement & 94 & 16874 & 9.85 & 37 & 11027 & 10.47 & 21 & 3668 & 1.95 & 17 & 3847 & 1.99 \\
\hline 1) SINES & 1 & 49 & 0.03 & 0 & 0 & 0 & 2 & 207 & 0.11 & 2 & 353 & 0.18 \\
\hline 2) LINES & 92 & 16749 & 9.77 & 33 & 9768 & 9.28 & 19 & 3461 & 1.84 & 13 & 2849 & 1.47 \\
\hline 3) LTR elements & 1 & 76 & 0.04 & 4 & 1259 & 1.20 & 0 & 0 & 0 & 2 & 645 & 0.33 \\
\hline DNA transposons & 4 & 203 & 0.12 & 0 & 0 & 0 & 5 & 137 & 0.07 & 8 & 1014 & 0.52 \\
\hline Unclassified & 0 & 0 & 0 & 0 & 0 & 0 & 0 & 0 & 0 & 2 & 330 & 0.17 \\
\hline \multirow[t]{2}{*}{ Total interspersed repeats } & & 17077 & 9.97 & & 5191 & 2.68 & & 3805 & 2.02 & & 5191 & 2.68 \\
\hline & \multicolumn{3}{|c|}{9 TGMCBA-217A3 } & \multicolumn{3}{|c|}{13 TGMCBA-136112 } & \multicolumn{3}{|c|}{17 TGMCBA-375I5 } & \multicolumn{3}{|c|}{17 TGMCBA-67H23 } \\
\hline$\% G C$ & & & 48.72 & & & 51.96 & & & 44.44 & & & 51.09 \\
\hline Satellites & 1 & 260 & 0.12 & 0 & 0 & 0 & 0 & 0 & 0 & 2 & 1074 & 0.64 \\
\hline Simple repeats & 65 & 3487 & 1.63 & 87 & 6437 & 3.8 & 41 & 1769 & 1.24 & 45 & 2772 & 1.66 \\
\hline Retroelement & 24 & 5688 & 2.66 & 51 & 11247 & 6.64 & 62 & 19590 & 13.73 & 44 & 11042 & 6.6 \\
\hline 1) SINES & 0 & 0 & 0 & 1 & 90 & 0.05 & 2 & 183 & 0.13 & 0 & 0 & 0 \\
\hline 2) LINES & 13 & 2373 & 1.11 & 34 & 5458 & 3.22 & 42 & 11979 & 8.4 & 32 & 6943 & 4.15 \\
\hline 3) LTR elements & 11 & 3315 & 1.55 & 16 & 5699 & 3.37 & 18 & 7428 & 5.21 & 12 & 4099 & 2.45 \\
\hline DNA transposons & 8 & 815 & 0.24 & 16 & 911 & 0.54 & 6 & 446 & 0.31 & 7 & 709 & 0.42 \\
\hline Unclassified & 0 & 0 & 0 & 3 & 316 & 0.19 & 1 & 97 & 0.07 & 0 & 0 & 0 \\
\hline \multirow[t]{2}{*}{ Total interspersed repeats } & & 6206 & 2.9 & & 12474 & 7.37 & & 20133 & 14.11 & & 11751 & 7.03 \\
\hline & \multicolumn{3}{|c|}{23 TGMCBA-227A15 } & \multicolumn{3}{|c|}{27 TGMCBA-23C5 } & \multicolumn{3}{|c|}{ Z CH261-129A16 } & \multicolumn{3}{|c|}{ Z TGMCBA-200J22 } \\
\hline$\% G C$ & & & 49.38 & & & 48.70 & & & 41.03 & & & 34.62 \\
\hline Satellites & 0 & 0 & 0 & 0 & 0 & 0 & 1 & 98 & 0.04 & 0 & 0 & 0 \\
\hline Simple repeats & 18 & 909 & 1.59 & 40 & 2247 & 1.11 & 68 & 2973 & 1.32 & 47 & 2450 & 1.45 \\
\hline Retroelement & 28 & 7991 & 14 & 161 & 47194 & 23.26 & 53 & 26179 & 11.59 & 46 & 16566 & 9.84 \\
\hline 1) SINES & 0 & 0 & 0 & 2 & 139 & 0.07 & 1 & 95 & 0.04 & 1 & 137 & 0.08 \\
\hline 2) LINES & 15 & 3180 & 5.57 & 117 & 25323 & 12.48 & 50 & 25215 & 11.16 & 32 & 11149 & 6.62 \\
\hline 3) LTR elements & 13 & 4811 & 8.43 & 42 & 21732 & 10.71 & 2 & 869 & 0.38 & 13 & 5280 & 3.14 \\
\hline DNA transposons & 1 & 85 & 0.15 & 6 & 979 & 0.48 & 12 & 2842 & 1.26 & 11 & 714 & 0.42 \\
\hline Unclassified & 0 & 0 & 0 & 0 & 0 & 0 & 0 & 0 & 0 & 0 & 0 & 0 \\
\hline Total interspersed repeats & & 8076 & 14.15 & & 48173 & 23.74 & & 29021 & 12.85 & & 17280 & 10.26 \\
\hline
\end{tabular}

$\mathrm{NE}$; number of elements

LO; length occupied

$\%$; sequence percentage

NKA2 and NKAW suggest that a large ancestral super-sex chromosome has strong correlation between squamate chromosome 2 (NKA2) and snake sex chromosomes. Alternatively, the shared partial synteny of amniote sex chromosomes could follow a stochastic pattern within a restricted set of species. It remains possible that the partial synteny of squamate chromosome 2 to several amniote sex chromosomes represents random homologies, given that only small sets of genes in a restricted set of species are involved. However, we also performed chromosome mapping using chicken and zebra finch BACs derived from other chromosomes and did not observe hybridization signals on NKA2, NKAZ, or NKAW. The same BACs were also used to perform chromosome mapping in other amniotes, which did not show hybridization signals such as repeat sequences ([36-38]; Singchat et al. unpublished data). Hence, mapping data on NKAW with several chicken and zebra finch BACs might not be random. However, 


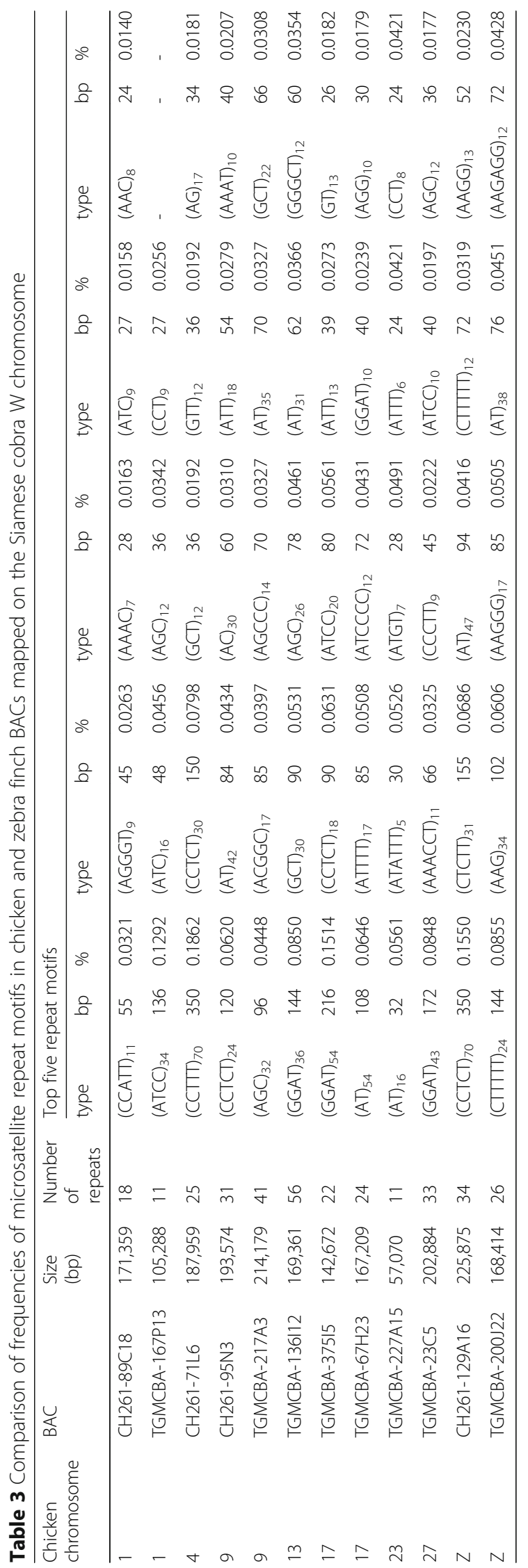


Table 4 Gene list relating to sex determination and sex developmental pathways for chicken and zebra finch BACs mapped on the Siamese cobra W chromosome

\begin{tabular}{ll}
\hline BAC & Gene \\
\hline CH261-89C18 & - \\
TGMCBA-167P13 & - \\
CH261-71L6 & DDX26B; gene on the X-chromosome \\
& Fith sex-bias in multiple tissues \\
& expression in testis than in ovary and \\
& ovotestis \\
CH261-95N3 & - \\
TGMCBA-217A3 & GPR149; novel oocyte-enriched gene, \\
& G-protein receptor 149 \\
& DHX36; genes may be involved in sex \\
& development, spermatogenesis and \\
TGMCBA-136112 & - \\
TGMCBA-37515 & - \\
TGMCBA-67H23 & - \\
TGMCBA-227A15 & - \\
TGMCBA-23C5 & - \\
CH261-129A16 & - \\
TGMCBA-200J22 & - \\
\hline
\end{tabular}

- : No data

limitations on the resolution of the FISH technique may indicate repeat sequences on NKAW, with repeats of other locations also escaping detection. At this stage, theories of independent rearrangements involving chromosomes $2, \mathrm{Z}$, and $\mathrm{W}$ in the snake lineage, and independent amplification of the same repeat sequences on snake and amniote sex chromosomes require further analysis of data from other squamate reptiles using FISH mapping and whole genome sequencing in order to investigate this hypothesis.

Sex-determining genes are well established in several amniotes, and orthologs or paralogs of the functional genes appear repeatedly in distantly related amniotes. This suggests the possibility of cooperative favorable linked sex chromosomes with hitchhiking effects (genes physically linked to one of the chosen sex-determining genes undergoing selective sweep) or interaction between genes [4]. The sequence of chicken and zebra finch BACs mapped to NKAW contained at least five known protein-coding genes involving sex determination and sex development pathways (Table 4). Two genes (GPR149 and DHX36) located on TGU9 BAC (TGMCBA-217A3) were mapped to NKAW as a twin dot hybridization signal. This hybridization pattern is unlikely to be caused by repeat sequences. However, we have no direct evidence that these genes are involved in snake sex determination or sex development. It is likely that convergent evolution of sex chromosomes across distantly related taxa led to genomic elements that are particularly adept in a sex-determining role. Are these genes coincidental, or are there sequences in these regions that have a selectable function in sex determination? More information regarding genomic analysis and transcriptomic activity from squamate reptiles is required to test this hypothesis.

\section{Conclusions}

This study suggests the fundamental basis on which the Siamese cobra chromosome 2 and the W sex chromosome share partial synteny and some characteristics of sex chromosomes observed in other amniotes, for example the amplification of repeat sequences. This in turn supports the hypothesis of ancestral super-sex chromosomes with overlaps of partial syntenies among amniote sex chromosomes. This might result from random distribution of microsatellite repeat motifs in each lineage as an alternative hypothesis, representing convergent sex chromosomal differentiation among amniote sex chromosomes. Further research is required to compare additional maps and sequences to explore syntenies in several squamate reptiles, using recent advances in comparative gene mapping and whole-genome sequencing. This may provide a more comprehensive understanding of the complexity of evolutionary sex determination and sex chromosomes in amniotes.

\section{Methods \\ Specimen Collection}

Two male and two female Siamese cobras (N. kaouthia) were collected from Snake Farm, Queen Saovabha Memorial Institute (QSMI, Bangkok). The sex of each individual was identified morphologically and confirmed using a molecular sexing approach [22-24]. Blood samples were collected from the ventral tail vein using a 23-gauge needle attached to 2-ml disposable syringes. These contained $10 \mathrm{mM}$ ethylenediaminetetraacetic acid (EDTA) for DNA extraction or 75 USP unit/ml heparin for cell culture. Whole genomic DNA was extracted following the standard salting-out protocol as described previously by Supikamolseni et al. [46] and used as templates for molecular sexing. DNA quality and concentration were determined using 1\% agarose gel electrophoresis and spectrophotometric analysis.

\section{Lymphocyte Cell culture and Chromosome Preparation}

Lymphocytes from two male and two female Siamese cobras were isolated from peripheral blood, and then cultured for 5 days in RPMI 1640 medium supplemented with 15\% fetal bovine serum (FBS), $3 \mu \mathrm{g} / \mathrm{ml}$ concanavalin A (type IV-S) (Sigma-Aldrich, St. Louis, MO, USA), $10 \mu \mathrm{g} / \mathrm{ml}$ lipopolysaccharide (Sigma-Aldrich), 1\% phytohaemagglutinin 


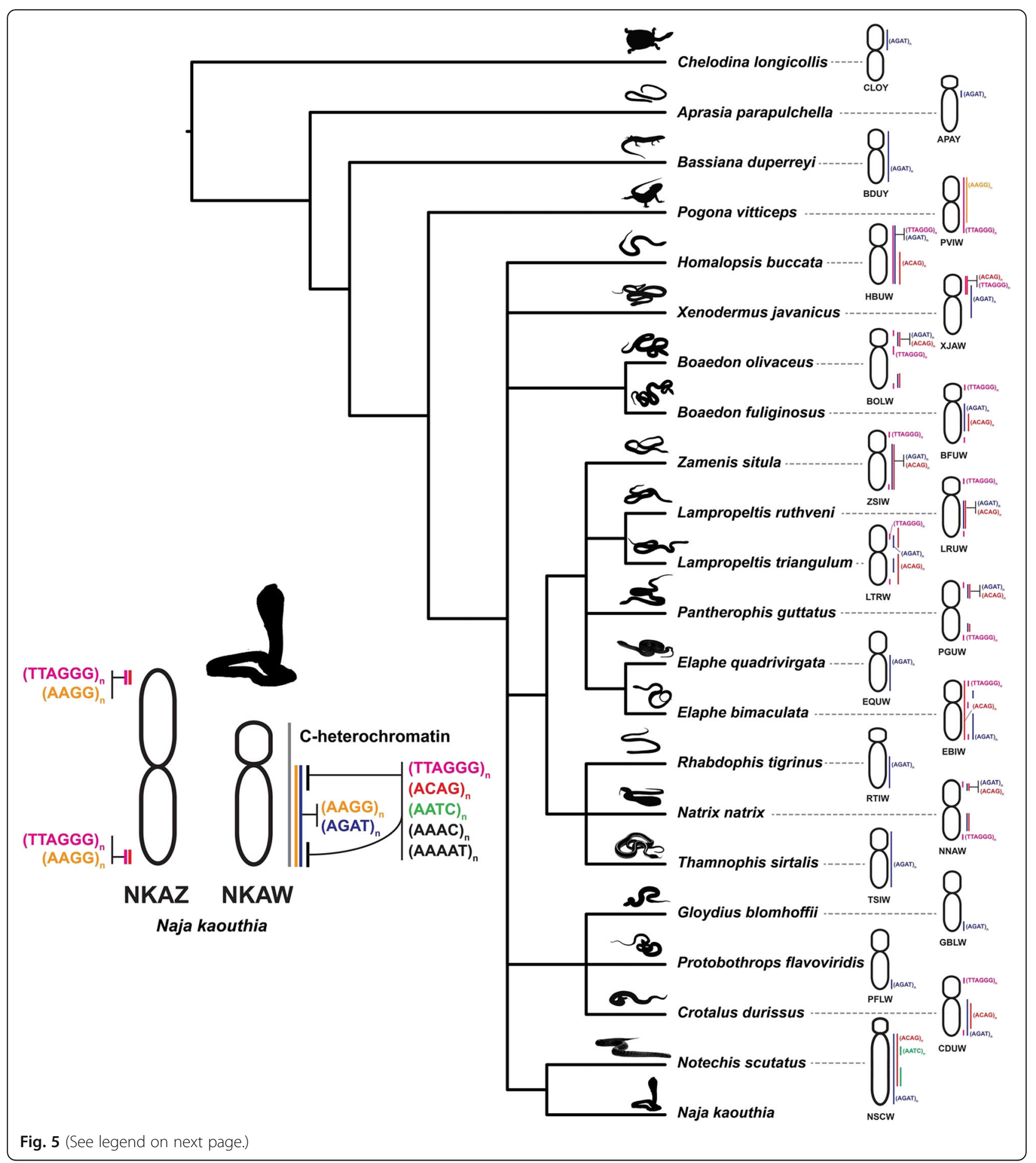


(See figure on previous page.)

Fig. 5 Schematic representation of microsatellite repeat motif amplification on the Siamese cobra W chromosome and other amniote sex chromosomes sharing the same motifs. Phylogeny was partially derived from Pyron et al. [43]. Regions where the microsatellite repeat motifs were hybridized are shown to the right of the chromosomes. Chromosome numbers indicate the pink-tailed worm-lizard (Aprasia parapulchella, APA), the Japanese striped snake (Elaphe quadrivirgata, EQU), the tiger keelback snake (Rhabdophis tigrinus, RTI), the habu Protobothrops flavoviridis, (PFL), the Japanese moccasin Gloydius blomhoffii, (GBL), the eastern three-lined skink (Bassiana duperreyi, BDU), the central bearded dragon (Pogona vitticeps, PVI), the eastern long-necked turtle (Chelodina longicollis, CLO), the tiger snake (Notechis scutatus, NSC), the tiger keelback snake (Rhabdophis tigrinus, RTI), the dragon snake (Xenodermus javanicus, XJA), the South American rattlesnake (Crotalus durissus, CDU), the masked water snake (Homalopsis buccata, HBU), the African house snake (Boaedon fuliginosus, BFU and Boaedon olivaceus, BOL), the milk snake (Lampropeltis triangulum, LTR), the Ruthven's kingsnake (Lampropeltis ruthveni, LRU), the corn snake (Pantherophis guttatus, PGU), the twinspotted ratsnake (Elaphe bimaculata), the leopard snake (Zamenis situla, ZSI), the grass snake (Natrix natrix, NNA), and the garter snake (Thamnophis sirtalis, TSI) showing shared microsatellite repeat motifs with the Siamese cobra W chromosome. Chromosomal locations of repeat motifs in the amniotes were obtained from the following sources: A. parapulchella from Matsubara et al. [53], E. quadrivirgata, R. tigrinus, P. flavoviridis and G. blomhoffii from Matsubara et al. [27, 28], B. duperreyi, P. vitticeps, C. longicollis, N. scutatus and R. tigrinus from Matsubara et al. [26, 27], X. javanicus from Rovatsos et al. [54], C. durissus, H. buccata, B. fuliginosus, B. olivaceus, L. triangulum, L. ruthveni, P. guttatus, E. bimaculata, Z. situla and N. natrix from Augstenová et al. [25], and T. sirtalis from Perry et al. [42]

(HA15) (Remel, Lenexa, KS, USA) and 1\% Antibiotic-Antimycotic (Life Technologies-Gibco, Carlsbad, CA, USA). After 5 days lymphocytes were subject to colcemid treatment $(100 \mathrm{ng} / \mathrm{ml})$ for $60 \mathrm{~min}$ and fixed (3:1 methanol/acetic acid) after hypotonic treatment in $0.075 \mathrm{M}$ $\mathrm{KCl}$, before being harvested. The cell suspension was dropped onto clean glass slides and air-dried. The slides were kept at $-80^{\circ} \mathrm{C}$ until required for use.

\section{Karyotyping and C-banding}

Morphology and size of macrochromosomes were characterized according to Turpin and Lejeune [47] and Levan et al. [48]. To examine the chromosomal distribution of constitutive heterochromatin, C-banding was performed using the standard barium hydroxide/saline/Giemsa method [49] with slight modification as follows: chromosome slides were treated with $0.2 \mathrm{~N} \mathrm{HCl}$ at room temperature for $60 \mathrm{~min}$ and then with $5 \% \mathrm{Ba}(\mathrm{OH})_{2}$ at 50 ${ }^{\circ} \mathrm{C}$ for $30 \mathrm{~s}$, followed by $2 \times \mathrm{SSC}$ at $65^{\circ} \mathrm{C}$ for $60 \mathrm{~min}$.

\section{Fluorescence in situ Hybridization (FISH) Mapping of Telomeric Repeat and Microsatellite Repeat Motifs} The chromosomal locations of telomeric (TTAGGG) sequences and 19 microsatellite repeat motifs: $(\mathrm{CA})_{15}$, $(\mathrm{GC})_{15},(\mathrm{GA})_{15},(\mathrm{AT})_{15},(\mathrm{CAA})_{10},(\mathrm{CAG})_{10},(\mathrm{CAT})_{10}$, $(\mathrm{CGG})_{10}, \quad(\mathrm{GAG})_{10}, \quad(\mathrm{AAT})_{10}, \quad(\mathrm{AAGG})_{8}, \quad(\mathrm{AATC})_{8}$, $(\mathrm{AGAT})_{8}, \quad(\mathrm{ACGC})_{8}, \quad(\mathrm{AAAT})_{8}, \quad(\mathrm{AAAC})_{8}, \quad(\mathrm{AATG})_{8}$, $(\text { AAATC })_{6}$, and $(\text { AAAAT })_{6}$ were determined using FISH as described previously $[16,50]$. We used commercially biotin-labeled 42-bp oligonucleotide complementary to $\left(\right.$ TTAGGG) ${ }_{n}$ sequences and 19 commercially biotin-labeled oligonucleotide microsatellite repeat probes (Macrogen Co., Seoul, Korea), ethanol-precipitated with salmon sperm DNA, and Escherichia coli tRNA. After the hybridization of biotin-labeled probes to the Siamese cobra chromosomes, the probes were detected by incubating the chromosome slides with avidin labeled with fluorescein isothiocyanate (avidin-FITC; Invitrogen, CA, USA).
Slides were subsequently stained with $1 \mu \mathrm{g} / \mathrm{ml}$ DAPI ( 4 ', 6'-diamidino-2-phenylindole). Fluorescence hybridization signals were captured using a cooled Charge-Coupled Device (CCD) camera mounted on a ZEISS Axioplan2 microscope and processed using MetaSystems ISIS v.5.2.8 software (MetaSystems, Altlussheim, Germany).

\section{Isolation, Amplification and Labeling of Chicken and Zebra finch Bacterial Artificial Chromosomes (BACs)} Chicken (Gallus gallus) and zebra finch (Taeniopygia guttata) BACs were applied for cross-species FISH mapping according to a range of the proportion of conserved elements shared across multiple species. Due to the high degree of apparent genome conservation between avian and reptilian species ([36-38]; Singchat et al. unpublished data), these sets of BACs were applied to the Siamese cobras. All BACs were anchored to the chicken and zebra finch genome assembly by linkage and sequencing. Twenty-six chicken and seventeen zebra finch BACs, comprising chicken chromosome 1 (GGA1), GGA2p, GGA4, GGA5, GGA9, GGA15, GGA17, GGA18, GGA23, GGA27 and GGAZ, and zebra finch chromosome 1 (TGU1B), TGU4A, TGU5, TGU9, TGU12, TGU13, TGU15, TGU17, TGU23, TGU27 and TGUZ, were isolated using the Qiagen Miniprep Kit (Qiagen, Manchester, UK) prior to amplification and direct labeling by nick translation (Roche, Welwyn Garden City, UK). Probes were labeled with Texas Red 12-dUTP (Invitrogen Corporation and Applied Biosystems Inc., Carlsbad, California, US) and FITC-12-UTP (Roche) prior to purification using the Qiagen Nucleotide Removal Kit (Qiagen).

\section{Cross-Species Bacterial Artificial Chromosome (BAC) Fluorescence in situ Hybridization (FISH) Mapping}

Chromosome slides were dehydrated through an ethanol series ( 2 min each in $2 \times$ SSC, $70 \%, 85 \%$, and $100 \%$ ethanol at room temperature). Probes were diluted in a 


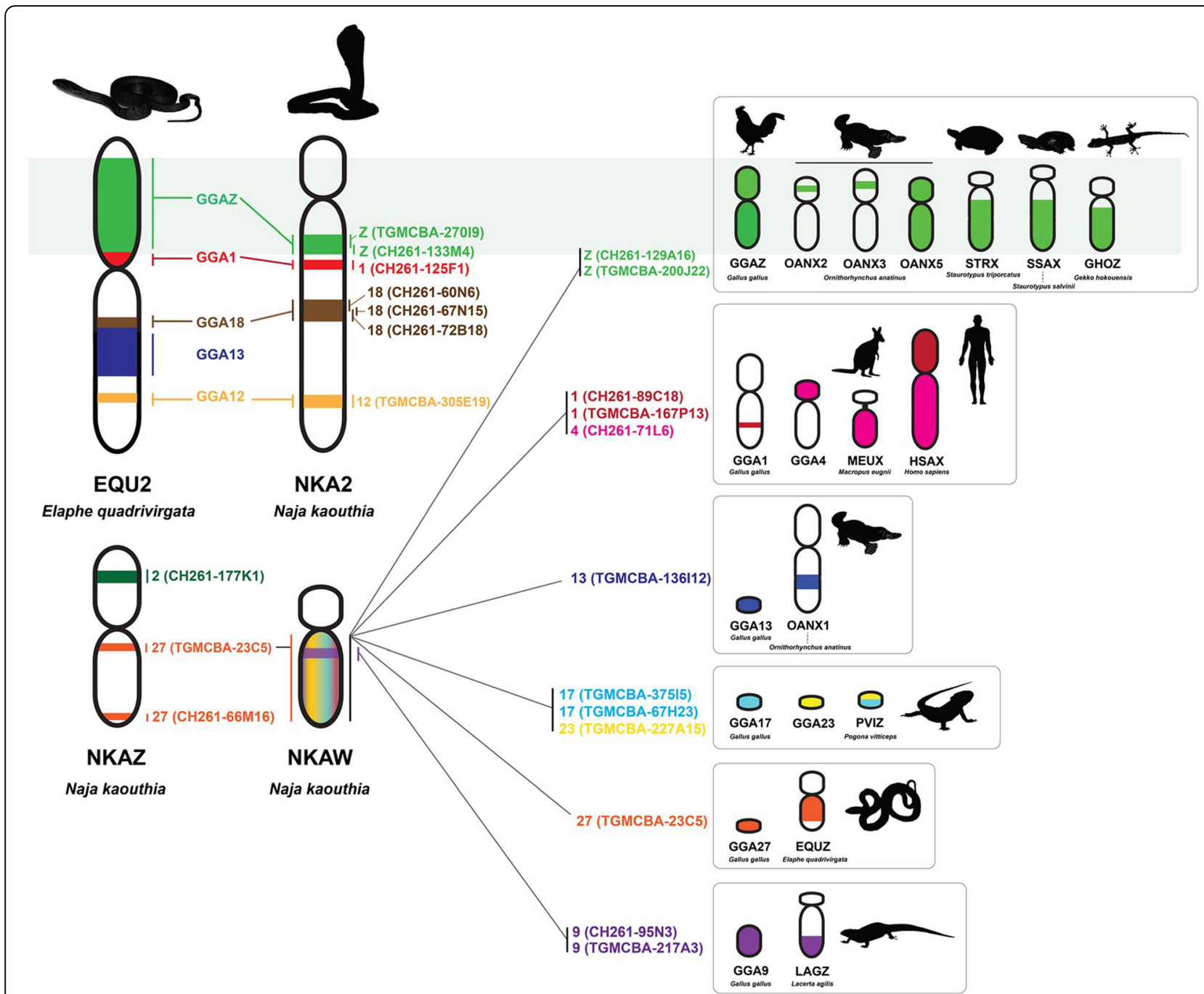

Fig. 6 Chromosome maps of the Siamese cobra chromosome 2, and Z and W chromosomes showing homologies with the chicken, zebra finch, and several other amniotes. This map was constructed using 43 BACs derived from the chicken and zebra finch. Locations of the BACs on Siamese cobra chromosomes are shown to the right of the chromosomes. Chromosome numbers indicate the chicken (Gallus gallus, GGA), the green anole (Anolis carolinensis, ACA), the bearded dragon lizard (Pogona vitticeps, PVI), the Japanese four-striped rat snake (Elaphe quadrivirgata, EQU), humans (Homo sapiens, HSA), the tammar wallaby (Macropus eugnii, MEU), the duck-billed platypus (Ornithorhynchus anatinus, OAN), the giant musk turtle (Staurotypus salvinii, SSA), the mexican musk turtle (Staurotypus triporcatus, STR), the Hokou gecko (Gekko hokouenesis, GHO), and the sand lizard (Lacerta agilis, LAG) showing partial homologies with the Siamese cobra chromosomes. Partial syntenies are shown using the same color. Chromosomal locations of genes in the amniotes were obtained from the following sources: G. gallus from Matsuda et al. [33], A. carolinensis from Alföldi et al. [12], E. quadrivirgata from Matsubara et al. [21], G. hokouenesis from Kawai et al. [10], P. vitticeps from Ezaz et al. [13], H. sapiens and M. eugnii from Grützner et al. [55], O. anatinus from Veyrunes et al. [15], S. salvinii from Kawagoshi et al. [5] S. triporcatus from Montiel et al. [56], and L. agilis from Srikulnath et al. [14]

Hybridization Solution I (Cytocell Ltd., Cambridge, UK) with Chicken Hybloc (Insight Biotechnology limited, Wembley, London, UK) and applied to the Siamese cobra chromosomes on a $37{ }^{\circ} \mathrm{C}$ hotplate before being sealed with rubber cement. Probe and target DNA were simultaneously denatured on a $75{ }^{\circ} \mathrm{C}$ hotplate for $2 \mathrm{~min}$ prior to hybridization in a humidified chamber at $37{ }^{\circ} \mathrm{C}$ for $72 \mathrm{~h}$. Slides were washed post-hybridization for $30 \mathrm{~s}$ in $2 \times \mathrm{SSC} / 0.05 \%$ Tween 20 at room temperature and then counterstained using VECTASHIELD antifade mounting medium with DAPI (Vector Laboratories, Inc., Burlingame, CA, USA). Images were captured using an Olympus BX61 epifluorescence microscope with a cooled CCD camera and SmartCapture system (Digital Scientific UK Ltd., Cambridge, UK). Confirmation of BAC signal order was achieved by dual color experiments where Texas Red 12-dUTP- and FITC-12-UTPlabeled probes were hybridized simultaneously. 


\section{Candidate Bacterial Artificial Chromosome (BAC) Sequence Analysis}

Gene position and gene structure in each candidate BAC were derived from ENSEMBL (ensembl.org/Gallus_gallus/Info/Annotation). The CpG content and repeat information were retrieved from the UCSC Genome Browser http://genome.ucsc.edu/ for the corresponding gene build. Simultaneously, each BAC sequence was subjected to homology search using Blastx and Blastn (RefSeq G. gallus and T. guttata). Repeat data were identified using Arian Smit's Repeat Masker program [51], which screens DNA sequences for interspersed repeats and low complexity DNA sequences. Query species were assumed to be Vertebrata, Metazoa, Repeat Masker Combined Database; Dfam_Consensus-20170127 and RepBase-20170127, run with rmblastn version 2.2.27+ [51]. Microsatellite repeat motifs were scanned from candidate BAC sequences using MSATCOMMANDER [52]. Parameters of the minimum number of repetitions were set at 10, 6, 4, 4, 4, and 4 for mono, di, tri, tetra, penta, and hexanucleotide motifs, respectively.

\section{Additional files}

Additional file 1: Figure S1. Chromosomal locations of microsatellite repeat motifs in the Siamese cobra (Naja kaouthia). Hybridization patterns of FITC-labeled (CA) 15 (a), (AT) 15 (b), (GC) 15 (c), (CAT) 10 (d), and (AAT) 10 (e) on DAPI-stained chromosomes. Scale bar represents $10 \mu \mathrm{m}$. (JPG $175 \mathrm{~kb}$ )

Additional file 2: Figure S2. Cytogenetic map of the Siamese cobra (Naja kaouthia), which shows chromosome homologies with chicken and zebra finch. This map was constructed with 25 chicken and zebra finch BACs mapped on the Siamese cobra macrochromosomes. Locations of BACs are shown to the right of the Siamese cobra chromosomes. The chromosome numbers show the chromosomes of the chicken (Gallus gallus, GGA) and zebra finch (Taeniopygia guttata, TGU), which show homologies with the Siamese cobra chromosomes. (JPG $118 \mathrm{~kb}$ )

Additional file 3: Table S1. Comparison of major classes of repeat sequences in chicken and zebra finch BACs mapped on the Siamese cobra Z chromosome. (DOCX $14 \mathrm{~kb}$ )

Additional file 4: Table S2. Comparison of frequencies of microsatellite repeat motifs in chicken and zebra finch BACs mapped on the Siamese cobra Z chromosome. (DOCX $13 \mathrm{~kb}$ )

Additional file 5: Table S3. Comparison of major classes of repeat sequences in chicken and zebra finch BACs mapped on the Siamese cobra chromosome 2. (DOCX $15 \mathrm{~kb}$ )

Additional file 6: Table S4. Comparison of frequencies of microsatellite repeat motifs in chicken and zebra finch BACs mapped on the Siamese cobra chromosome 2. (DOCX $14 \mathrm{~kb}$ )

Additional file 7: Table S5. Comparison of major classes of repeat sequences in 22 chicken and zebra finch BACs mapped on the Siamese cobra chromosome 1 and microchromosomes. (DOCX $23 \mathrm{~kb}$ )

Additional file 8: Table S6. Comparison of frequencies of microsatellite repeat motifs in 22 chicken and zebra finch BACs mapped on the Siamese cobra chromosome 1 and microchromosomes. (DOCX 19 kb)

\section{Abbreviations}

ACA: Anolis carolinensis; APA: Aprasia parapulchella; BACs: bacterial artificial chromosomes; BDU: Bassiana duperreyi; BFU: Boaedon fuliginosus; Bkm: banded krait minor satellite; BOL: Boaedon olivaceus; CCD: Charge-Coupled Device; CDU: Crotalus durissus; CLO: Chelodina longicollis; DAPI: 4', 6-diamidino-2- phenylindole; EBI: Elaphe bimaculata; EQU: Elaphe quadrivirgata; FBS: fetal bovine serum; FISH: Fluorescent in situ hybridization; FITC: Fluorescein isothiocyanate; GBL: Gloydius blomhoffii; GGA: Gallus gallus; GHO: Gekko hokouenesis; HBU: Homalopsis buccata; HSA: Homo sapiens; LAG: Lacerta agilis; LRU: Lampropeltis ruthveni; LTR: Lampropeltis triangulum; MEU: Macropus eugnii; NKA: Naja kaouthia; NNA: Natrix natrix; NSC: Notechis scutatus:

OAN: Ornithorhynchus anatinus; PFL: Protobothrops flavoviridis; PGU: Pantherophis guttatus; PVI: Pogona vitticeps; QSMI: Queen Saovabha Memorial Institute; RTI: Rhabdophis tigrinus; SSA: Staurotypus salvinii; STR: Staurotypus triporcatus; TGU: Taeniopygia guttata; TSI: Thamnophis sirtalis; XJA: Xenodermus javanicus; ZSI: Zamenis situla

\section{Acknowledgments}

We would like to thank Tanapong Tawan, Taksa Vasaruchapong and Panithi Laoungbua (Snake Farm, Queen Saovabha Memorial Institute, Thai Red Cross Society, Thailand), and Lucas Kiazim (University of Kent, UK), and the Conservation Research and Education Division, Zoological Park Organization, Dusit, Bangkok, Thailand for help with sample collection.

\section{Funding}

This study was financially supported by grants from the Thailand Research Fund (TRF; no. RSA6180075, PHD6010014, PHD6010082, MSD6010035) awarded to KS, WS, AS and PT, the Thailand Research Fund - Newton Fund Placement, Travel Grant for PhD Supervisors (GA/PhD/Sup/Year4/003) awarded to KS, Kasetsart University Research and Development Institute (KURDl; grant no. 80.60) awarded to KS, the Fellowship of Capacity Building for Kasetsart University on Internationalization at Kasetsart University (No.0513.10109/8384) awarded to WA and KS, the Center for Advanced Studies in Tropical Natural Resources, National Research University-Kasetsart University (CASTNAR, NRU-KU, Thailand) awarded to KS, the National Research Council of Thailand (NRCT; No. 2560096003012) awarded to SB and KS, the Center of Excellence on Agricultural Biotechnology, Science and Technology Postgraduate Education and Research Development Office, Office of Higher Education Commission, Ministry of Education (AG-BIO/ PERDO-CHE) awarded to KS. ROC was supported by Biotechnology and Biological Sciences Research Council (BBSRC) grant (BB/K008161/1) awarded to DK.

\section{Availability of data and materials}

The datasets used and/or analyzed during the current study are available from the corresponding author on reasonable request.

\section{Authors' contributions}

W.S. and K.S. drafted the manuscript; W.S., R.O., D.G., and K.S. conceived the ideas and designed methodology; W.S., R.O., P.T., A.S., and K.S. carried out the lab work; W.S. and K.S. participated in data analysis; W.S., R.O., P.T., A.S., S.S., S.S., N.M., S.B., S.P., L.C., D.G., and K.S. reviewed the data and the manuscript. All authors gave final approval for publication.

Ethics approval and consent to participate

Animal care and all experimental procedures were approved by the Animal Experiment Committee, Kasetsart University, Thailand (approval no. ACKU59 -SCI-034 and ACKU61-SCI-024) and conducted in accordance with the Regulations on Animal Experiments at Kasetsart University.

\section{Consent for publication}

Not applicable.

\section{Competing interests}

The authors declare that they have no competing interests.

\section{Publisher's Note}

Springer Nature remains neutral with regard to jurisdictional claims in published maps and institutional affiliations.

\section{Author details}

'Laboratory of Animal Cytogenetics and Comparative Genomics (ACCG), Department of Genetics, Faculty of Science, Kasetsart University, Bangkok 10900, Thailand. ${ }^{2}$ Animal Breeding and Genetics Consortium of Kasetsart University (ABG-KU), Bangkok 10900, Thailand. ${ }^{3}$ School of Biosciences, University of Kent, Canterbury CT2 7NY, United Kingdom. ${ }^{4}$ Department of Research and Development, Queen Saovabha Memorial Institute, The Thai Red Cross Society, Bangkok 10330, Thailand. ${ }^{5}$ Department of Fishery Biology, 
Faculty of Fisheries, Kasetsart University, Bangkok 10900, Thailand. ${ }^{6}$ Bureau of Conservation and Research, Zoological Park Organization under the Royal Patronage of His Majesty the King, Bangkok, Thailand. ${ }^{7}$ Department of Biology, Faculty of Science, Naresuan University, Phitsanulok 65000, Thailand. ${ }^{8}$ Snake Farm, Queen Saovabha Memorial Institute, The Thai Red Cross Society, Bangkok 10330, Thailand. ${ }^{9}$ Center for Advanced Studies in Tropical Natural Resources, National Research University-Kasetsart University, Kasetsart University, Bangkok 10900, Thailand. ${ }^{10}$ Center of Excellence on Agricultural Biotechnology (AG-BIO/PERDO-CHE), Bangkok 10900, Thailand.

\section{Received: 29 August 2018 Accepted: 21 November 2018} Published online: 17 December 2018

\section{References}

1. Charlesworth B. The evolution of sex chromosomes. Science. 1991;251:1030-3.

2. Bachtrog D, Mank JE, Peichel CL, et al. Sex Determination: why so many ways of doing it? PLoS Biol. 2014;12:e1001899.

3. Charlesworth D, Charlesworth B, Marais G. Steps in the evolution of heteromorphic sex chromosomes. Heredity (Edinb). 2005;95:118-28.

4. Ezaz T, Srikulnath K, Graves JA. Origin of amniote sex chromosomes: an ancestral super-sex chromosome, or common requirements? J Hered. 2017;108:94-105.

5. Kawagoshi T, Uno Y, Nishida C, Matsuda Y. The Staurotypus Turtles and Aves share the same origin of sex chromosomes but evolved different types of heterogametic sex determination. PLoS One. 2014;9:e105315.

6. Kawagoshi T, Uno Y, Matsubara K, Matsuda Y, Nishida C. The ZW micro-sex chromosomes of the Chinese soft-shelled turtle (Pelodiscus sinensis, Trionychidae, Testudines) have the same origin as chicken chromosome 15. Cytogenet Genome Res. 2009;125:125-31.

7. Kawagoshi T, Nishida C, Matsuda Y. The origin and differentiation process of $X$ and $Y$ chromosomes of the black marsh turtle (Siebenrockiella crassicollis, Geoemydidae, Testudines). Chromosome Res. 2012;20:95-110.

8. Badenhorst D, Stanyon R, Engstrom T, Valenzuela N. A ZZ/ZW microchromosome system in the spiny softshell turtle, Apalone spinifera, reveals an intriguing sex chromosome conservation in Trionychidae. Chromosome Res. 2013;21:137-47.

9. Montiel EE, Badenhorst D, Tamplin J, Burke RL, Valenzuela N. Discovery of the youngest sex chromosomes reveals first case of convergent co-option of ancestral autosomes in turtles. Chromosoma. 2016;126:105-13.

10. Kawai A, Ishijima J, Nishida C, et al. The ZW sex chromosomes of Gekko hokouenesis (Gekkonidae, Squamata) represent highly conserved homology with those of avian species. Chromosoma. 2009;118:43-51.

11. Srikulnath K, Uno Y, Nishida C, Ota H, Matsuda Y. Karyotype reorganization in the Hokou gecko (Gekko hokouenesis, Gekkonidae): the process of microchromosome disappearance in Gekkota. PLoS One. 2015;10:e0134829.

12. Alföldi J, Palma FD, Grabherr M, et al. The genome of the green anole lizard and a comparative analysis with birds and mammals. Nature. 2011;477:587.

13. Ezaz T, Azad B, O'Meally D, et al. Sequence and gene content of a large fragment of a lizard sex chromosome and evaluation of candidate sex differentiating gene R-spondin 1. BMC Genomics. 2013;14:899.

14. Srikulnath K, Matsubara K, Uno Y, Nishida C, Olsson M, Matsuda Y. Identification of the linkage group of the $Z$ sex chromosomes of the sand lizard (Lacerta agilis, Lacertidae) and elucidation of karyotype evolution in lacertid lizards. Chromosoma. 2014;123:563-75.

15. Veyrunes F, Waters PD, Miethke P, et al. Bird-like sex chromosomes of platypus imply recent origin of mammal sex chromosomes. Genome Res. 2008;18:965-73.

16. Srikulnath $\mathrm{K}$, Matsubara $\mathrm{K}$, Uno Y, et al. Karyological characterization of the butterfly lizard (Leiolepis reevesii rubritaeniata, Agamidae, Squamata) by molecular cytogenetic approach. Cytogenet Genome Res. 2009;125:213-23.

17. Srikulnath K, Nishida C, Matsubara K, et al. Karyotypic evolution in squamate reptiles: comparative gene mapping revealed highly conserved linkage homology between the butterfly lizard (Leiolepis reevesii rubritaeniata, Agamidae, Lacertilia) and the Japanese four-striped rat snake (Elaphe quadrivirgata, Colubridae, Serpentes). Chromosome Res. 2009;17:975-86.

18. Srikulnath K, Uno Y, Nishida C, Matsuda Y. Karyotype evolution in monitor lizards: cross-species chromosome mapping of cDNA reveals highly conserved synteny and gene order in the Toxicofera clade. Chromosome Res. 2013;21:805-19.

19. Pokorná $M$, Giovannotti $M$, Kratochvíl $L$, et al. Strong conservation of the bird $Z$ chromosome in reptilian genomes is revealed by comparative painting despite 275 million years divergence. Chromosoma. 2011;120:455-68.
20. Gamble T, Castoe TA, Nielsen SV, et al. The discovery of XY sex chromosomes in a Boa and Python. Curr Biol. 2017;27:2148-2153.e4.

21. Matsubara K, Tarui H, Toriba M, et al. Evidence for different origin of sex chromosomes in snakes, birds, and mammals and step-wise differentiation of snake sex chromosomes. Proc Natl Acad Sci U S A. 2006;103:18190-5.

22. Laopichienpong N, Muangmai N, Chanhome L, et al. Evolutionary dynamics of the gametologous CTNNB1 gene on the $Z$ and $W$ chromosomes of snakes. J Hered. 2017;108:142-51.

23. Laopichienpong $N$, Tawichasri $P$, Chanhome $L$, et al. A novel method of caenophidian snake sex identification using molecular markers based on two gametologous genes. Ecol Evol. 2017;7:4661-9.

24. Tawichasri P, Laopichienpong N, Chanhome $L$, et al. Using blood and noninvasive shed skin samples to identify sex of caenophidian snakes based on multiplex PCR assay. Zool Anz. 2017;271:6-14.

25. Augstenová B, Mazzoleni S, Kratochvíl L, Rovatsos M. Evolutionary dynamics of the W chromosome in caenophidian snakes. Genes. 2018;9:5.

26. Matsubara K, O'Meally D, Azad B, et al. Amplification of microsatellite repeat motifs is associated with the evolutionary differentiation and heterochromatinization of sex chromosomes in Sauropsida. Chromosoma. 2015a;125:111-23.

27. Matsubara K, Uno Y, Srikulnath K, Matsuda Y, Miller E, Olsson M. No interstitial telomeres on autosomes but remarkable amplification of telomeric repeats on the $\mathrm{W}$ sex chromosome in the sand lizard (Lacerta agilis). J Hered. 2015b;106:753-7.

28. Matsubara K, Nishida C, Matsuda Y, Kumazawa Y. Sex chromosome evolution in snakes inferred from divergence patterns of two gametologous genes and chromosome distribution of sex chromosome-linked repetitive sequences. Zool Lett. 2016;2:19.

29. Oguiura N, Ferrarezzi H, Batistic RF. Cytogenetics and molecular data in snakes: a phylogenetic approach. Cytogenet Genome Res. 2009;127:128-42.

30. O'Meally D, Patel HR, Stiglec R, et al. Non-homologous sex chromosomes of birds and snakes share repetitive sequences. Chromosome Res. 2010;18:787-800.

31. Epplen JT, McCarrey JR, Sutou S, Ohno S. Base sequence of a cloned snake W-chromosome DNA fragment and identification of a male-specific putative mRNA in the mouse. Proc Natl Acad Sci U S A. 1982;79:3798-802.

32. Vicoso B, Emerson JJ, Zektser Y, Mahajan S, Bachtrog D. Comparative sex chromosome genomics in snakes: differentiation, evolutionary strata, and lack of global dosage compensation. PLoS Biol. 2013;11:e1001643.

33. Matsuda Y, Nishida-Umehara C, Tarui H, et al. Highly conserved linkage homology between birds and turtles: bird and turtle chromosomes are precise counterparts of each other. Chromosome Res. 2005;13:601-15.

34. Matsubara K, Kuraku S, Tarui H, et al. Intra-genomic GC heterogeneity in sauropsids: evolutionary insights from cDNA mapping and GC (3) profiling in snake. BMC Genomics. 2012;13:604.

35. Olmo E, Signorino G. Chromorep: a reptile chromosomes. database. 2005; http://chromorep.univpm.it. Accessed 26 July 2018.

36. Damas J, O'Connor R, Farré $M$, et al. Upgrading short-read animal genome assemblies to chromosome level using comparative genomics and a universal probe set. Genome Res. 2017;27:875-84.

37. O'Connor RE, Romanov MN, Kiazim LG, et al. Reconstruction of the diapsid ancestral genome permits chromosome evolution tracing in avian and nonavian dinosaurs. Nat Commun. 2018;9:1883.

38. O'Connor RE, Farré M, Joseph S, et al. Chromosome-level assembly reveals extensive rearrangement in saker falcon and budgerigar, but not ostrich, genomes. Genome Biol. 2018;19:171.

39. Graves JAM. Sex chromosome specialization and degeneration in mammals. Cell. 2006;124:901-14.

40. Schartl M, Schmid M, Nanda I. Dynamics of vertebrate sex chromosome evolution: from equal size to giants and dwarfs. Chromosoma. 2016;125:553-71.

41. Young MJ, O'Meally D, Sarre SD, Georges A, Ezaz T. Molecular cytogenetic map of the central bearded dragon, Pogona vitticeps (Squamata: Agamidae). Chromosome Res. 2013;21:361-74.

42. Perry BW, Card DC, McGlothlin JW, et al. Molecular adaptations for sensing and securing prey and insight into amniote genome diversity from the garter snake genome. Genome Biol Evol. 2018;10:2110-29.

43. Rovatsos M, Altmanová M, Pokorná JM, Augstenová B, Kratochvíl L. Cytogenetics of the Javan file snake (Acrochordus javanicus) and the evolution of snake sex chromosomes. J Zool Syst Evol Res. 2018:56:117-25.

44. Pyron RA, Burbrink FT, Wiens JJ. A phylogeny and revised classification of Squamata, including 4161 species of lizards and snakes. BMC Evol Biol. 2013;13:93 
45. Steinemann S, Steinemann M. Retroelements: tools for sex chromosome evolution. Cytogenetic Genom Res. 2005;110:134-43.

46. Supikamolseni A, Ngaoburanawit N, Sumontha M, et al. Molecular barcoding of venomous snakes and species-specific multiplex PCR assay to identify snake groups for which antivenom is available in Thailand. Genet Mol Res. 2015;14:13981-97.

47. Turpin R, Lejeune J. Les Chromosomes Humains. Paris: Gauthier-Villars; 1965.

48. Levan A, Fredga K, Sandberg A. Nomenclature for centromeric position on chromosomes. Hereditas. 1964;52:201-20.

49. Sumner AT. A simple technique for demonstrating centromeric heterochromatin. Exp Cell Res. 1972;75:304-6.

50. Matsuda Y, Chapman VM. Application of fluorescence in situ hybridization in genome analysis of the mouse. Electrophoresis. 1995;16:261-72.

51. Smit AFA, Hubley R, Green P. RepeatMasker Open-4.0. 2015. http://www. repeatmasker.org. Accessed 26 July 2018.

52. Faircloth BC. MSATCOMMANDER: detection of microsatellite repeat arrays and automated, locus-specific primer design. Mol Ecol Resour. 2008:8:92-4

53. Matsubara K, Knopp T, Sarre SD, Georges A, Ezaz T. Karyotypic analysis and FISH mapping of microsatellite motifs reveal highly differentiated XX/XY sex chromosomes in the pink-tailed worm-lizard (Aprasia parapulchella, Pygopodidae, Squamata). Mol Cytogenet. 2013;6:60.

54. Rovatsos M, Johnson Pokorná M, Kratochvíl L. Differentiation of sex chromosomes and karyotype characterisation in the dragonsnake Xenodermus javanicus (Squamata: Xenodermatidae). Cytogenet. Genome Res. 2015;147:48-54.

55. Grützner F, Rens W, Tsend-Ayush E, et al. In the platypus a meiotic chain of ten sex chromosomes shares genes with the bird $Z$ and mammal $X$ chromosomes. Nature. 2004;432:913-7.

56. Montiel EE, Badenhorst D, Lee LS, Literman R, Trifonov V, Valenzuela N. Cytogenetic insights into the evolution of chromosomes and sex determination reveal striking homology of turtle sex chromosomes to amphibian autosomes. Cytogenet Genome Res. 2016;148:292-304.

Ready to submit your research? Choose BMC and benefit from:

- fast, convenient online submission

- thorough peer review by experienced researchers in your field

- rapid publication on acceptance

- support for research data, including large and complex data types

- gold Open Access which fosters wider collaboration and increased citations

- maximum visibility for your research: over $100 \mathrm{M}$ website views per year

At $\mathrm{BMC}$, research is always in progress.

Learn more biomedcentral.com/submissions 